\title{
ERRATUM
}

\section{Erratum to: Morphological Variability in Leaves and Molecular Characterization of Novel Table Grape Candidate Cultivars (Vitis vinifera L.)}

\author{
Vittorio Alba - Carlo Bergamini - Maria Francesca Cardone • \\ Marica Gasparro • Rocco Perniola • \\ Rosalinda Genghi · Donato Antonacci
}

Published online: 7 May 2014

(C) Springer Science+Business Media New York 2014

\section{Erratum to: Mol Biotechnol}

DOI 10.1007/s12033-013-9729-6

Table 7 of the original version of the article unfortunately contains an error. The presentation of values in Table 7 was incorrect and the corrected table is given below.

The online version of the original article can be found under doi:10.1007/s12033-013-9729-6.

\footnotetext{
V. Alba - C. Bergamini - M. F. Cardone - M. Gasparro ·

R. Perniola $\cdot$ R. Genghi · D. Antonacci $(\square)$

Consiglio per la Ricerca e la sperimentazione in Agricoltura -

CRA, Unità di ricerca per l'uva da tavola e la vitivinicoltura

in ambiente mediterraneo, Via Casamassima 148,

70010 Turi, BA, Italy

e-mail: donato.antonacci@entecra.it

V. Alba

e-mail: vittorio.alba@libero.it

C. Bergamini

e-mail: carlo.bergamini@entecra.it

M. F. Cardone

e-mail: mariafrancesca.cardone@entecra.it

M. Gasparro

e-mail: marica.gasparro@entecra.it

R. Perniola

e-mail: rocco.perniola@entecra.it

R. Genghi

e-mail: rosalinda.genghi@entecra.it
} 
Table 7 Allele size in relative base pair distance to allele size $n$ as coded by OIV descriptors list of twenty-six table grape genotypes at six microsatellite loci

\begin{tabular}{|c|c|c|c|c|c|c|c|c|c|c|c|c|}
\hline \multirow[b]{3}{*}{ 89IxU119 } & \multirow{2}{*}{\multicolumn{2}{|c|}{$\frac{\text { VVS2 }}{\text { OIV801 }}$}} & \multirow{2}{*}{\multicolumn{2}{|c|}{$\frac{\text { VVMD5 }}{\text { OIV802 }}$}} & \multirow{2}{*}{\multicolumn{2}{|c|}{$\frac{\text { VVMD7 }}{\text { OIV803 }}$}} & \multirow{2}{*}{\multicolumn{2}{|c|}{$\frac{\text { VVMD27 }}{\text { OIV804 }}$}} & \multirow{2}{*}{\multicolumn{2}{|c|}{$\frac{\text { VrZAG62 }}{\text { OIV805 }}$}} & \multirow{2}{*}{\multicolumn{2}{|c|}{$\frac{\text { VrZAG79 }}{\text { OIV806 }}$}} \\
\hline & & & & & & & & & & & & \\
\hline & 12 & 26 & 12 & 16 & 16 & 24 & 4 & 20 & 18 & 30 & 18 & 22 \\
\hline Alzey real & 16 & 20 & 10 & 14 & 8 & 8 & 10 & 10 & 14 & 30 & 14 & 14 \\
\hline Bellini & 28 & 32 & 6 & 16 & 8 & 16 & 4 & 10 & 14 & 18 & 2 & 24 \\
\hline Cardinal n. & 12 & 12 & 4 & 14 & 18 & 18 & 4 & 10 & 12 & 12 & 14 & 18 \\
\hline Ceresa & 10 & 26 & 6 & 18 & 18 & 18 & 10 & 20 & 12 & 20 & 10 & 14 \\
\hline Conegliano 218 & 10 & 28 & 14 & 16 & 16 & 18 & 4 & 20 & 12 & 18 & 18 & 20 \\
\hline Dalmasso VII-6 & 10 & 20 & 4 & 6 & 8 & 18 & 4 & 10 & 20 & 30 & 18 & 18 \\
\hline Dalmasso XIII-11 & 12 & 14 & 16 & 16 & 12 & 16 & 4 & 6 & 14 & 20 & 18 & 22 \\
\hline Dalmasso XXIII-12 & 12 & 14 & 4 & 16 & 16 & 18 & 4 & 6 & 18 & 30 & 18 & 22 \\
\hline Delizia di Vaprio & 10 & 10 & 4 & 10 & 18 & 20 & 20 & 20 & 12 & 30 & 10 & 20 \\
\hline Gargiulo 102011 & 12 & 26 & 6 & 12 & 8 & 18 & 4 & 6 & 12 & 14 & 10 & 18 \\
\hline Gargiulo 88086 & 26 & 28 & 6 & 12 & 8 & 18 & 6 & 20 & 14 & 30 & 10 & 20 \\
\hline Gargiulo 88435 & 10 & 12 & 14 & 14 & 18 & 20 & 10 & 18 & 12 & 26 & 14 & 20 \\
\hline Gargiulo 90393 & 12 & 22 & 14 & 16 & 12 & 18 & 10 & 18 & 12 & 14 & 20 & 20 \\
\hline IxES 21 & 10 & 12 & 4 & 10 & 12 & 18 & 4 & 19 & 12 & 30 & 18 & 18 \\
\hline IxFT 87 & 10 & 26 & 10 & 12 & 8 & 12 & 4 & 10 & 14 & 30 & 14 & 18 \\
\hline ISV IxV93 & 10 & 28 & 6 & 16 & 8 & 16 & 4 & 10 & 18 & 20 & 2 & 18 \\
\hline Italia & 10 & 26 & 10 & 16 & 12 & 16 & 4 & 19 & 18 & 30 & 18 & 20 \\
\hline Pirovano 100 & 10 & 14 & 6 & 16 & 18 & 18 & 6 & 19 & 28 & 30 & 8 & 20 \\
\hline Pirovano 244 & 10 & 12 & 10 & 14 & 16 & 18 & 4 & 19 & 18 & 30 & 20 & 22 \\
\hline Pirovano 432 & 10 & 26 & 10 & 16 & 18 & 18 & 4 & 4 & 12 & 30 & 18 & 18 \\
\hline Pirovano 727 & 12 & 26 & 6 & 10 & 8 & 16 & 6 & 19 & 14 & 18 & 20 & 20 \\
\hline Prosperi 190 & 12 & 26 & 10 & 16 & 8 & 16 & 10 & 19 & 14 & 18 & 6 & 20 \\
\hline Sugraone & 12 & 12 & 4 & 14 & 8 & 18 & 4 & 6 & 12 & 14 & 18 & 22 \\
\hline Unterkofler 50 & 10 & 20 & 4 & 16 & 20 & 32 & 4 & 8 & 20 & 30 & 10 & 18 \\
\hline Valsesia 54 & 10 & 12 & 10 & 18 & 16 & 18 & 2 & 18 & 18 & 30 & 18 & 20 \\
\hline$H_{O}{ }^{\mathrm{a}}$ & 0.88 & & 0.92 & & 0.81 & & 0.88 & & 0.96 & & 0.77 & \\
\hline$H_{E}^{\mathrm{b}}$ & 0.81 & & 0.84 & & 0.76 & & 0.82 & & 0.81 & & 0.79 & \\
\hline $\mathrm{Na}^{\mathrm{c}}$ & 9 & & 7 & & 7 & & 8 & & 7 & & 9 & \\
\hline $\mathrm{NDG}^{\mathrm{d}}$ & 13 & & 16 & & 11 & & 14 & & 13 & & 15 & \\
\hline
\end{tabular}

a Observed heterozygosity

b Expected heterozygosity

${ }^{c}$ Observed number of alleles

${ }^{d}$ Number of different genotypes

Homozygous loci are reported in bold 